\title{
Identifying critical success factors in EDA industry using DEMATEL method
}

\author{
Chia-Chi Sun* \\ Assistant Professor, Grade Institute \& Department of International Business, Tamkang University \\ Email: samsun0712@gmail.com; ccsun@mail.tku.edu.tw
}

Received 12 June 2012; Accepted 23 March 2014

\begin{abstract}
Electronic design automation (EDA) is a category of software tools for designing electronic systems such as printed circuit boards and integrated circuits. However, the EDA industry has been locked into virtually flat growth for the past several years. Time-to-market pressures and design complexity are critical challenges that EDA industry face today. With this background, this paper attempts to identify and analyze the Critical Success Factors (CSFs) in EDA industry. This research proposes the decision making trial and evaluation laboratory (DEMATEL) as the main analytical tool. The DEMATEL can be used as an effective method to handle the inner dependences within a set of criteria. Results show that the critical local demand condition and government are the causal competitive advantage factors for the EDA industry and could have the significant role in responding to the EDA industry. This paper draws on the research results for managerial practice implications and suggests some empirical tactics to enhance management performance for the EDA industry.
\end{abstract}

Keywords: EDA industry; Diamond mode; Critical Success Factors; Decision Making Trial and Evaluation Laboratory (DEMATEL); Mu1tiple criteria decision-making (MCDM).

\section{Introduction}

Electronic design automation (EDA) is a category of software tools for designing electronic systems such as printed circuit boards and integrated circuits. The tools work together in a design flow that chip designers use to design and analyze entire semiconductor chips ${ }^{1}$. The EDA industry has been locked into virtually flat growth for the past several years ${ }^{2}$. Average selling prices are declining as pressure for discounting grows and customers increasingly regard EDA as a tactical, rather than strategic, purchase. The EDA industry is on a path to gradual decay and irrelevance unless a dramatic shift takes place ${ }^{2}$. The problem is not that EDA has changed, or that EDA tools and services no longer offer value. The problem is that the electronics industry has changed its value proposition from silicon to software applications, changing dramatically the demands placed upon EDA customers. While electronic products in the past were sold on a one time basis, with no revenue recognition after the sale, Apple pioneered a new business model that allows it to recognize revenue from 'apps' that are sold continuously. In EDA, providers must not only help customers reduce design and verification costs and solves technology problems, but also help them differentiate themselves from competitors, and collaborate with partners worldwide. To do this, the industry must focus on integrated solutions. Time-to-market pressures and design complexity are critical challenges that design teams face today. The EDA will, therefore, have to sharpen the focus on cost effective verification process and design, and on IP integration to ensure design predictability ${ }^{3}$. The demand for incorporation of more functionality into the products is driving complexity. With this background, this paper attempts to identify and analyze the Critical Success Factors (CSFs) in EDA industry.

*No.151, Yingzhuan Rd., Tamsui Dist., New Taipei City 25137, Taiwan R.O.C. 
CSFs can be defined as those areas in which satisfactory results lead to successful competitive performance ${ }^{1}$. The CSF approach has been widely adopted and used in a variety different field of study to determine key factors which are essential to the success of any program or technique. Critical success factors are complex activities necessary in the study of disruptive innovations ${ }^{4,5}$. A relatively high number of parameters must be considered to create a complete picture of the market. By definition, multi-criteria analysis is a very good candidate method for dealing with this type of complex problem. Multiple criteria decision-making (MCDM) methods imply a modeling activity, which should clarify many aspects, making the decision process more transparent $6,7,8,9$.

This paper demonstrates an integrated Multiple criteria decision making (MCDM) technique that is more appropriate for identifying critical success factors in EDA industry. The MCDM method has high potential to reduce the cost and time to create an appropriate framework for solving problems. To solve the managerial issue, the study applies the Decision Making Trial and Evaluation Laboratory (DEMATEL) 10,11

This study regards the evaluation index system for identifying critical success factors in EDA industry as a system that includes causal relationships between the influencing factors, which is a new way to solve critical success factors in EDA industry problem. Interactions of factors which influence critical success factors in EDA industry are very complicated. Application of recessive knowledge instead of evident knowledge is therefore required to make a reliable decision. The DEMATEL methodology can exploit recessive knowledge such as expert experiences and instincts. This methodology uses the least resource devotion, focuses on the core driving factor in the system and the improvement direction for optimal critical success factors in EDA industry. Considering the impact of causal relationships between influencing factors helps to assess the importance of influencing factors and uses the least resource devotion to solve a complicated problem more reasonably. Knowledge of these relationships provides the organization with the required decision making information. Therefore, we demonstrate that the DEMATEL multiple criteria evaluation technique is more appropriate than many traditional methods because it provides practitioners with a valuable tool for use to solve critical success factors in EDA industry problems. Through this well-constructed DEMATEL approach our research results show that a proper operations strategy and management of both resource usage and task arrangement in accordance with policy making will accomplish excellent strategic alliance for the EDA industry. Through this well-constructed DEMATEL approach our research results show that a proper operations strategy and management of both resource usage and task arrangement in accordance with policy making will accomplish competitive advantages for the EDA industry.

We present a multi-criteria model comprised of the preferences of various dimensions first. Secondly, we introduce a tool, DEMATEL, to analyze the critical success factors of EDA industry. Thirdly, we provide some preliminary insights into the EDA industry based on empirical evidence collected from the main actors in this industry. It is a suitable method that helps in gathering group knowledge to form a structural model, as well as in visualizing the casual relationship between the sub-systems through a casual diagram ${ }^{12}$. The judgment of decision-makers is often given as crisp values. However, crisp values are an inadequate reflection of the vagueness in the real world ${ }^{13}$. Should the adopted value $p$ be too low, the structure of the system will remain complex and difficult to understand, while if threshold value $p$ is too high, the structure would be oversimplified and important influences ignored. The threshold values have not standard settings. The thresholds are decided through expert options in this field. This study applied DEMATEL to address this issue.

The primary objective of this study is to address the following research questions:

(1) To confirm and validate the critical success factors of EDA industry

(2) To examine the relationship between critical success factors

This study uses the Taiwan EDA industry as the case study. The relationship between the different factors used to make a casual map that identifies the casual group and effect group is discussed. EDA industry operators are provided with some strategic recommendations based on the research results. The remainder of this paper is as follows. Section 2 presents the methodology, DEMATEL. Section 3 introduces the research design, which includes the research framework, 
research procedure, sample selection and the empirical results. Some empirical research is discussed in Section 4. Concluding remarks are given in Section 5 .

\section{Assessment dimensions for EDA Industry}

In this section, we review the theoretical background to this paper. A major breakthrough for the cluster concept was Porter's Competitive Advantage of Nations (1998) which advocated specialization according to historical strength by emphasizing the power of industrial clusters. We believe that most of the above chiefly issue from a major breakthrough for the industrial analysis concept derived from Porter's Competitive Advantage of Nations ${ }^{14}$, which advocated specialization according to historical strength by emphasizing the power of industrial clusters. Porter highlighted that multiple factors beyond those internal to the firm may improve its performance. In his "diamond model," four sets of interrelated forces are brought forward to explain industrial clusters: factor input conditions; local demand conditions; related and supported industries; and firm structure, strategy, and rivalry. Embracing more vivid and straightforward dimensions comparing to other approaches, the diamond model is therefore deemed our most fitting analytic framework while we are pursuing effective measurement of industrial analysis from the viewpoint of EDA industry competitive advantages ${ }^{15}$.

\subsection{Factor Conditions}

Porter agreed that a state's or nation's endowment of factors for encouraging production has a role in determining competitive advantage. However, Porter broadened the definition of factors for production into five major categories: human resources, physical resources, knowledge resources, capital resources, and infrastructure ${ }^{16,17}$.

Abundant natural resources, which are factors of production, could provide the original momentum for establishing an industry ${ }^{3}$. Their presence might also have enticed a predecessor industry to the location, thereby creating the initial framework for a subsequent industry ${ }^{14}$.

The fact that competitive pressure compels firms to innovate in order to overcome their microeconomic environment's disadvantages represents a major theme in Porter's work. The remaining fundamental determinants in the model play an important and powerful role in inciting firms to innovate so as to remain competitive players in their industries. Specialized factors of production are skilled labor, capital, and information infrastructure. Specialized factors involve heavy, sustained investment, and they are more difficult to duplicate. These factors include entrepreneurship and venture capital.

Entrepreneurship is the engine of cluster development growth. Entrepreneurs play an important role in selecting and applying new ideas, and venture capital investment plays a vital role in creating phenomenal economic growth. Finally, having a science and information infrastructure means that a country or region establishes a science and technology platform for improving or upgrading science linkage between players, such as science parks or information centers. The science park concept was originated in the late 1950s. The idea was, and still is, to provide a technical, logistical, administrative, and financial infrastructure to help young enterprises gain a toehold for their products in an increasingly competitive market.

\subsection{Local Demand Conditions}

Consumer demand plays possibly the most important role in forming and building up an industrial cluster ${ }^{5}$. A large number of industrial customers in the nearby area create sufficient demand to enable suppliers to acquire and operate expensive specialized machinery.

Porter ${ }^{14}$ has argued that a sophisticated domestic market is an important element for producing competitiveness. Firms that face a sophisticated domestic market are likely to sell superior products because the market demands high quality, and a close proximity to such consumers enables the firm to better understand the needs and desires of the customers ${ }^{18}$. As a result, demand conditions can stimulate an industry through local demand for a product that also proves viable in regional, national, and international markets.

\subsection{Related and Supporting Industries}

Spatial proximity of upstream or downstream industries facilitates the exchange of information and promotes a continuous exchange of ideas and innovations ${ }^{19}$. The availability, density, and interconnectedness of vertically and horizontally related industries are important drivers for industrial clusters ${ }^{18}$. This includes suppliers and related industries.

Related industries refer to firms that provide complementary products or services to one another. 
While competing on the basis of their value chain management within their product- or service-specific industry, they might share or coordinate certain activities, such as distribution, technology development, manufacturing, or marketing ${ }^{14,20}$. Competitive related industries can provide opportunities for technological exchanges and, possibly, can accelerate the development of competitive local supplier industries serving both. However, close working relationships among related industries do not happen automatically. Related industries must explicitly seek to forge alliances that will add to their competitive advantage ${ }^{17}$.

Close working relationships between downstream firms and local input suppliers can facilitate the process of innovation and upgrading ${ }^{14}$. Related and supporting industries could drive the creation of an industry through spin-offs, serving a particular market that is outside the realm of another local industry ${ }^{17}$.

\subsection{Firm Structure, Strategy, and Rivalry}

Porter ${ }^{14}$ argues that intense competition spurs innovation. The world is dominated by dynamic conditions. Direct competition impels firms to work for increases in productivity and innovation. Firm strategy, structure, and rivalry refer to the various approaches to a firm's inception, organization, and management that establish the context for local rivalry and competitive advantage ${ }^{21}$. Differences in management systems and organizational structure offer opportunities for establishing competitive advantage. Relationships between labor and management represent a particularly important element for the firm, given their powerful impact on the process of innovation and improvements

14. Porter established that rivalry with domestic firms proves to be more beneficial than other factors in terms of innovation and improvements. Local rivals compel one another to seek effective cost-cutting measures, product/service innovations, and organizational improvements. Local competitive pressure also leads to commercially successful firms, which in turn, lures new firms to the industry.

Rivalry is the key element compelling the initial industry to become competitive through upgrading and innovation. A cluster of domestic rivals encourages the formation of more specialized suppliers and related industries. The geographic proximity in the cluster between rival firms and their suppliers or related industries facilitates research exchanges and collaboration. This broadens the depth, breadth, and specialization of the cluster, thereby inducing further investment in advanced infrastructure and factor creation $^{17}$

\subsection{Government Support}

The role of government in Porter's Diamond Model is to act as a catalyst and challenger; it is to encourage - or even push-companies to raise their aspirations and move to higher levels of competitive performance. Government must encourage companies to raise their performance, to stimulate early demand for advanced products, to focus on specialized factor creation, and to stimulate local rivalry by limiting direct cooperation and enforcing anti-trust regulations.

Besides, government must provide the required infrastructural needs of the developing industrial cluster. The role of the government in a regional economy is necessarily a variable over the life cycle of the industry cluster, and as a result it needs to have the capability to identify and monitor the set of natural industries that exist within the region and their stages of development 14. However, government's role requires a paradigm shift, both in "mind set" and in programs and services 14. In the new paradigm government acts as facilitator, promoting partnerships and alliances, and focusing on investments in skills development, infrastructure, and new technologies. The government has taken a much more commanding role than formerly, encouraging the creation of clusters by offering inducements to companies to relocate there.

\subsection{Chance}

Chances are occurrences that are outside of control of a firm. They are important because they create discontinuities in which some gain competitive positions and some lose 22 .

Overall, the above-mentioned theoretical models contributed to our understanding of critical success factors of EDA industry. These studies hint at a set of potentially relevant factors driving competitive advantage of EDA industry. At the same time, it is obvious that there is a research gap in regards to multicriteria analysis on critical success factors and in developing an understanding of the cause-effect relationship of complex social science problems. With this background, this paper attempts to identify and analyze the Critical Success Factors (CSFs) in EDA 
industry. Thus, this research proposes a decision making trial and evaluation laboratory (DEMATEL) as the main analytical tool. Below we discuss the research method, DEMATEL in detail.

\section{The Decision Making Trial and Evaluation Laboratory (DEMATEL) Method}

This is a method that helps gather group knowledge to form a structural model, as well as visualizing the casual relationship between the sub-systems through a casual diagram. The judgment of decision-makers is often given as crisp values. However, crisp values are an inadequate reflection of the vagueness in the real world. This study applied DEMATEL to address this issue. This study regards the evaluation index system for strategic alliance as a system that includes causal relationships between the influencing factors, which is a new way to solve that problem. The factors that influence critical success factors in EDA industry are so complicated that recessive knowledge is more reliable than evident knowledge to make a decision. The DEMATEL methodology can exploit recessive knowledge such as expert experiences and instincts. This methodology uses the least resource devotion, focuses on the core driving factor in the system and the improvement direction, on which we can choose the optimal strategic pattern. Considering the impact of a causal relationship can more reasonably assess the importance of influencing factors and use the least resources devoted to solving a complicated problem when there are causal relationships between the influencing factors, which effectively and accurately provide the organization with the required decision making information. Therefore, we demonstrate that the DEMATEL multiple criteria evaluation techniques are more appropriate than the traditional method and provide practitioners with a valuable tool to solve these problems.

The DEMATEL method was developed to study the structural relations in a complex system ${ }^{7,23}$. The mathematical concepts are borrowed from Liou, Yen, and Tzeng ${ }^{7}$ and $\mathrm{Wu}^{24}$. DEMATEL has been adapted in many academic fields, such as industry analysis. $\mathrm{Wu}$ and Lee ${ }^{12}$ combined DEMATEL and fuzzy theory to segment required competencies for better promoting the competency development of global managers. Liou, Yen and Tezng ${ }^{7}$ used fuzzy logic and DEMATEL to build an effective safety management system for airlines. Huang, Shyu, and Tzeng ${ }^{25}$ used DEMATEL and gray relational analysis to reconfigure innovation policy portfolios presented for the Taiwanese government's policy definition.

The DEMATEL model construction process is described below:

Step 1: Generating the direct-relation matrix

To measure the relationship between criteria requires that the comparison scale be constructed according to the following four levels: No influence (0), Low influence (1), Medium influence (2), High influence (3), and Very high influence (4). The integer score that the $k$ th expert gives to indicate the degree that factor $i$ has on factor $j$ is $x_{i j}^{k}{ }^{7}$. The $n \times n$ matrix $A$ is found by averaging all experts' scores.

$$
a_{i j}=\frac{1}{H} \sum_{k=1}^{H} x_{i j}^{k}
$$

Step 2: Normalizing the direct-relation matrix

On the base of the direct-relation matrix $A$, the normalized direct-relation matrix $X$ can be obtained by the following formulas:

$$
\text { Let } s=\max \left(\max _{I f i \notin n} \sum_{j=1}^{n} a_{i j}, \max _{1 \neq j \neq n} \sum_{i=1}^{n} a_{i j}\right)
$$

Then $X=\frac{A}{S}$

The sum of each row $j$ of matrix $A$ represents the direct effects that factor $i$ gives to the other factors; $\max \left(\max _{\text {lfifn }} \sum_{j=1}^{n} a_{i j}, \max _{l f j \neq n} \sum_{i=1}^{n} a_{i j}\right)$ represents the direct effects on others.

Step 3: Attaining the total-relation matrix

Once the normalized direct-relation $X$ is obtained, the total-relation matrix $T$ can be calculated by applying the following formula, (4), in which $I$ is denoted as the identity matrix ${ }^{23,24}$.

$$
T=X(I-X)^{-1}
$$

Step 4: Producing a causal diagram

The sum of rows and the sum of columns are separately denoted as vector $\quad D$ and vector $R$. The horizontal axis vector $(D+R)$, named "Prominence," is made by adding $D$ to $R$, which represents how much importance the criterion has. Equally, the vertical axis $(D-R)$, named "Relation," is made by subtracting $D$ from $R$, which may divide 
criteria into a cause group and an effect group. Based on the above statements, when $(D-R)$ is positive, the criterion belongs to the cause group. Otherwise, when the $(D-R)$ is negative, the criterion belongs to the effect group. Therefore, the causal diagram can be acquired by mapping the dataset of ${ }^{12}(D+R, D-R)$.

$$
\begin{gathered}
T=\left[t_{i j}\right]_{n \times n} i, j=1,2, \ldots, n \\
D=\left[\sum_{j=1}^{n} t i j\right]=\left[t_{i \square}\right]_{n \times 1}, i=1, \ldots n \\
R=\left[\sum_{i=1}^{n} t_{i j}\right]_{l \times n}^{t}=\left[t_{g j}\right]_{n \times 1}, j=1, \ldots n
\end{gathered}
$$

Step 5: Setting a threshold value and obtaining the inner dependence matrix

To explain the structural relation among factors while keeping the complexity of the whole system at a manageable level, it is necessary to set a threshold value $p$ to filter out negligible effects in matrix $T$. Only the factors whose effect in matrix $T$ is greater than the threshold value will be shown in an inner dependence matrix. Here the threshold value $p$ has been chosen by the experts and the results of the literature review. In order to explain the structural relation between the factors, it is necessary to decide a threshold value to remove the some unsuitable effects from consideration in matrix $T$. At this stage, experts will discuss how to decide each factor's threshold to make the rational decisions. The threshold values have not standard settings. The thresholds are decided through expert options in this field.

The DEMATEL methodology can exploit recessive knowledge to the full such as expert experiences and instincts. The decision maker can obtain a better understanding of the interrelationship in the selection model and also the importance ranking of each evaluation dimension and criterion. DEMATEL designs and arranges the system structural model using expert knowledge. It mainly helps the managers to reduce the interference of too much information among numerous influential factors. DEMATEL has also been adapted in many academic fields, such as industry analysis. Wu and Lee ${ }^{44}$ combined DEMATEL and fuzzy theory to segment required competencies for better promoting the competency development of global managers. Liou et al. ${ }^{7}$ used fuzzy logic and Decision Making Trial and Evaluation Laboratory to build an effective safety management system for airlines. Huang et al. ${ }^{25}$ applied DEMATEL Model to reconfigure innovation policy portfolios. Tzeng et al. ${ }^{26}$ addressed the independent relations of evaluation criteria with the aid of factor analysis and the dependent relations of evaluation criteria with the aid of DEMATEL. Wu ${ }^{24}$ applied the ANP and DEMATEL approach to help companies that need to evaluate and select KM strategies.

\section{Empirical Study and Discussion}

This paper identifies and analyzes the Critical Success Factors of EDA industry. Six dimensions were identified based on a comprehensive review of the literature and interviews with both academic and practitioners. A DEMATEL method was applied to analyze and classify these dimensions. DEMATEL helps decision makers to explore the casual relationships among the identified factors. In this section, an empirical study is presented to illustrate the application how EDA Industry applied this proposed method to enhance their advantages.

\subsection{Selecting the committee of experts who have experienced about this research issue}

Ten experts were invited to evaluate the criteria. We anticipated the interview would last two hours. The first part consisted of a presentation of the previous results (i.e., first MCDM model). The objective was to refresh memories and open a discussion. This presentation is based on a report distributed to the experts before starting the interview. We wanted the experts to have a common understanding of the results before the mobile payment evaluation. This analysis was conducted using an asynchronous mode with the experts. We collected empirical data from several key experts involved in different industries. The industry representation was optimal as we covered all relevant industry sectors (IC design, IC foundry, EDA). A great majority of the experts participated in the previous interview campaign. Therefore, they were already quite familiar with our approach. The committee followed the proposed method with a four-step procedure. The questionnaire survey was conducted from May 2009 to July 2010.

\subsection{Developing the evaluation dimensions}

Through the literature investigation and expert opinions, the committee adopted 6 dimensions. The evaluation dimensions are Factor Conditions $\left(\mathrm{D}_{1}\right)$, Local Demand 
Conditions $\left(\mathrm{D}_{2}\right)$, Related and Supporting Industries $\left(\mathrm{D}_{3}\right)$, Firm Structure, Strategy, and Rivalry, Government Support $\left(D_{5}\right)$ and Chance $\left(D_{6}\right)$. Based on the above dimensions, the study employed the DEMATEL method for capturing the complex relationships among these evaluation criteria. The hierarchical structure of this research decision problem is shown in Figure 1.

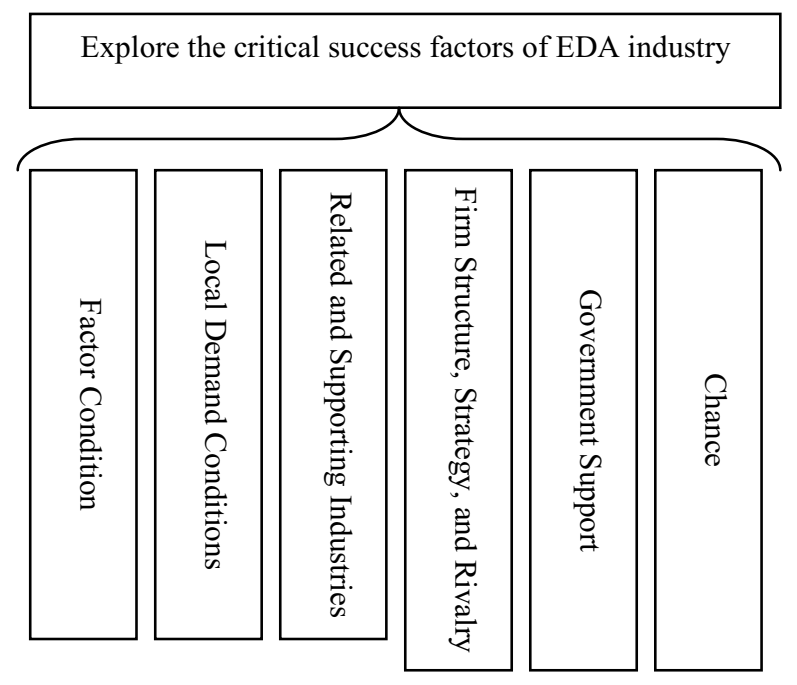

Figure 1 Research Framework

\subsection{Identify Relationship between Factors Using DEMATEL}

Our purpose is not only to find out the most important Critical Success Factors for the competitive advantage of EDA industry but also to measure the relationships among these Critical Success Factors. These experts followed our proposed method with a three-step procedure.

First, we made the decision goal and set up a committee. Through literature investigation and studying experts' opinions, the committee finally adopted six dimensions. More specifically, an expert validity survey was designed for the invited experts to evaluate the criteria by providing their suggestions and revisions. It is believed that a panel of experts can further enhance the quality of the chosen criteria. The purpose of the interviews was to obtain more specific ideas about the evaluation criteria from experienced incluster patricians' points of view. Based on the significant viewpoints received from the interviews, we confirmed the expert validity of the preliminary criteria. As noted, we adopted the factors of the Diamond Model 14 in addition to the cultural concept as our Critical Success Factors. Table 1 entails the details of their definitions. Based on the above dimensions, we employed the DEMATEL method for capturing the complex relationships among these evaluation dimensions.

Table 1 The Critical Success Factors of EDA Industry

\begin{tabular}{c|c|l}
\hline \hline \multicolumn{2}{c|}{ Dimension } & \multicolumn{1}{c}{ Dimension Description } \\
\hline \hline $\begin{array}{c}\text { Factor } \\
\text { Conditions }\end{array}$ & $\mathrm{D}_{1}$ & $\begin{array}{l}\text { Specialized factors of production are skilled labor, capital, and information infrastructure. The } \\
\text { factors include entrepreneurship and venture capital. Entrepreneurship is the engine of cluster } \\
\text { development growth. Venture capital markets create a positive impact on industrial development. } \\
\text { The science and information infrastructure means that a country or region should establish a science } \\
\text { and technology platform for improving or upgrading science linkage between players, such as } \\
\text { science parks or information centers. }\end{array}$ \\
\hline $\begin{array}{c}\text { Local Demand } \\
\text { Conditions }\end{array}$ & $\mathrm{D}_{2}$ & $\begin{array}{l}\text { Consumer demand plays an important role in forming and building up an industrial cluster. A large } \\
\text { market also enables suppliers to provide specialized products and services. } \\
\text { Suppliers gain from the nearby market for their output, while the client firms in the cluster gain from } \\
\text { easy access to a range of services. }\end{array}$ \\
\hline $\begin{array}{c}\text { Related and } \\
\text { Supporting } \\
\text { Industries }\end{array}$ & $\mathrm{D}_{3}$ & $\begin{array}{l}\text { Spatial proximity of upstream or downstream industries facilitates the exchange of information and } \\
\text { promotes a continuous exchange of ideas and innovations. The availability, density, and } \\
\text { interconnectedness of vertically and horizontally related industries are an important driver for } \\
\text { industrial clusters. }\end{array}$ \\
\hline $\begin{array}{c}\text { Firm } \\
\text { Structure, } \\
\text { Strategy, and } \\
\text { Rivalry }\end{array}$ & $\mathrm{D}_{4}$ & $\begin{array}{l}\text { Intense competition spurs innovation. The world is dominated by dynamic conditions. Direct } \\
\text { competition impels firms to work for increases in productivity and innovation. }\end{array}$ \\
\hline
\end{tabular}




\begin{tabular}{c|c|l}
\hline $\begin{array}{c}\text { Government } \\
\text { Support }\end{array}$ & $\mathrm{D}_{5}$ & $\begin{array}{l}\text { Government must provide the required infrastructural needs for a developing industrial cluster. } \\
\text { Government also encourages companies to raise their performance, to stimulate early demand for } \\
\text { advanced products, and to focus on specialized factor creation; government can stimulate local } \\
\text { rivalry by limiting direct cooperation and enforcing anti-trust regulations. }\end{array}$ \\
\hline Chance & $\mathrm{D}_{6}$ & $\begin{array}{l}\text { Chance events are occurrences that are outside of control of a firm. They are important because they } \\
\text { create discontinuities in which some gain competitive positions and some lose. }\end{array}$ \\
\hline \hline
\end{tabular}

This paper identifies and analyzes the critical factors of critical success factors for EDA industry. Six dimensions were identified based on a comprehensive review of the literature and interviews with both academic and practitioners.

\section{Step 1: Generating the assessments of decision - makers}

Then we obtained the collected pair-wise comparison results; the preliminary average direct-influence matrix is shown in Table 2.

Table 2 Direct-Influence Matrix

\begin{tabular}{l|cccccc}
\multicolumn{7}{c}{ Table 2 Direct-Influence Matrix } \\
\hline & $\mathrm{D}_{1}$ & $\mathrm{D}_{2}$ & $\mathrm{D}_{3}$ & $\mathrm{D}_{4}$ & $\mathrm{D}_{5}$ & $\mathrm{D}_{6}$ \\
\hline $\mathrm{D}_{1}$ & 0.00 & 0.10 & 1.90 & 2.70 & 1.00 & 0.50 \\
$\mathrm{D}_{2}$ & 0.30 & 0.00 & 1.60 & 1.20 & 1.40 & 1.20 \\
$\mathrm{D}_{3}$ & 1.50 & 1.50 & 0.00 & 1.90 & 1.20 & 1.00 \\
$\mathrm{D}_{4}$ & 2.20 & 0.30 & 1.50 & 0.00 & 1.20 & 0.90 \\
$\mathrm{D}_{5}$ & 2.20 & 0.40 & 1.90 & 1.10 & 0.00 & 1.20 \\
$\mathrm{D}_{6}$ & 0.40 & 0.60 & 0.60 & 0.70 & 0.90 & 0.00 \\
\hline
\end{tabular}

Step 2: Normalizing the direct-relation fuzzy matrix Equations (2)-(3) were used to calculate the normalized direct-relation matrix as following Table 3:

Table 3 Normalized Direct-Relation Matrix

\begin{tabular}{c|cccccc}
\hline & $\mathrm{D}_{1}$ & $\mathrm{D}_{2}$ & $\mathrm{D}_{3}$ & $\mathrm{D}_{4}$ & $\mathrm{D}_{5}$ & $\mathrm{D}_{6}$ \\
\hline $\mathrm{D}_{1}$ & 0.000 & 0.014 & 0.268 & 0.380 & 0.141 & 0.070 \\
$\mathrm{D}_{2}$ & 0.042 & 0.000 & 0.225 & 0.169 & 0.197 & 0.169 \\
$\mathrm{D}_{3}$ & 0.211 & 0.211 & 0.000 & 0.268 & 0.169 & 0.141 \\
$\mathrm{D}_{4}$ & 0.310 & 0.042 & 0.211 & 0.000 & 0.169 & 0.127 \\
$\mathrm{D}_{5}$ & 0.310 & 0.056 & 0.268 & 0.155 & 0.000 & 0.169 \\
$\mathrm{D}_{6}$ & 0.056 & 0.085 & 0.085 & 0.099 & 0.127 & 0.000 \\
\hline
\end{tabular}

Step 3: Attaining the total-relation matrix Once the normalized direct-relation is obtained the total-relation matrix $\tilde{T}$ can be calculated as following Table 4.

Table 4 Total-Influence Matrix

\begin{tabular}{l|cccccc}
\hline & $\mathrm{D}_{1}$ & $\mathrm{D}_{2}$ & $\mathrm{D}_{3}$ & $\mathrm{D}_{4}$ & $\mathrm{D}_{5}$ & $\mathrm{D}_{6}$ \\
\hline $\mathrm{D}_{1}$ & 1.0579 & 0.4720 & 1.2687 & 1.4192 & 0.9311 & 0.7406 \\
$\mathrm{D}_{2}$ & 0.9475 & 0.4051 & 1.1011 & 1.1021 & 0.8776 & 0.7473 \\
$\mathrm{D}_{3}$ & 1.2767 & 0.6567 & 1.1307 & 1.4079 & 1.0153 & 0.8511 \\
$\mathrm{D}_{4}$ & 1.2470 & 0.4734 & 1.1920 & 1.0902 & 0.9196 & 0.7561 \\
$\mathrm{D}_{5}$ & 1.3265 & 0.5277 & 1.3163 & 1.3148 & 0.8428 & 0.8461 \\
$\mathrm{D}_{6}$ & 0.5950 & 0.3144 & 0.6290 & 0.6648 & 0.5367 & 0.3586 \\
\hline
\end{tabular}

\section{Step 4: Producing a causal diagram}

Then we used formula (5)-(7) as based on the normalized matrix to produce the causal diagram by mapping a dataset of $(D+R, D-R)$, as displayed in Figure 2. the Tables 5 means total effects and net effects for each factor respectively.

Table 5Total Effects and Net Effects for Each Factor

\begin{tabular}{ccccc}
\hline & $\mathrm{D}$ & $\mathrm{R}$ & $\mathrm{D}+\mathrm{R}$ & $\mathrm{D}-\mathrm{R}$ \\
\hline $\mathrm{D} 1$ & 5.890 & 6.451 & 12.340 & -0.561 \\
$\mathrm{D} 2$ & 5.181 & 2.849 & 8.030 & 2.331 \\
$\mathrm{D} 3$ & 6.338 & 6.638 & 12.976 & -0.299 \\
$\mathrm{D} 4$ & 5.678 & 6.999 & 12.677 & -1.321 \\
$\mathrm{D} 5$ & 6.174 & 5.123 & 11.297 & 1.051 \\
$\mathrm{D} 6$ & 3.099 & 4.300 & 7.398 & -1.201 \\
\hline
\end{tabular}

We can then clearly and intuitionally discover the importance order of these dimensions for driving industrial cluster growth, according to the resulting causal diagram. Also looking at this causal diagram, these driving forces can be divided into a causal group, 
which includes $\mathrm{D}_{2}$ and $\mathrm{D}_{5}$, along with an effect group, which includes $\mathrm{D}_{1}, \mathrm{D}_{3}, \mathrm{D}_{4}$, and $\mathrm{D}_{6}$.

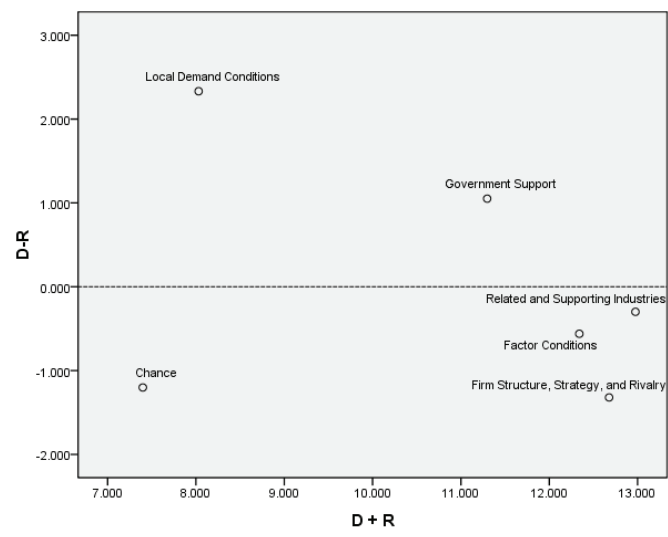

Figure2 Impact-relationship map

\section{Conclusions and Remarks}

This paper employed the DEMATEL method to analyze the model and considered suggestions as the key successes factors in EDA industry. First, this research helps divide all dimensions into causal groups and effect groups in terms of the key successes factors behind EDA industry. According to our research results, local demand conditions and government support are the major causal driving forces for the growth of EDA industry.

The local demand condition is an important EDA industry driver. Proximity to local market plays a key role in potentially increasing a firm's competitive advantage. Timeframes tend to be shorter for responding to local demand pressures, and the firms tend to be more confident in understanding and satisfying local demand requests. Porter indicated that local market size is most important for enhancing competitive advantage in industries with substantial research and development requirements, major economies of scale in production, or significant generational leaps in technology (Porter 1998). A rapidly growing local demand provides firms with the incentive to incorporate new technologies faster and to upgrade or expand their capacity with the necessary conviction that they will be used. This pulling force for innovations from the demand side has its complementary pushing force located among related and supporting industries ${ }^{27}$.

Government support will improve the competitive advantage of EDA industry. Governments need to take a more pro-active approach to: 1) promote collaboration among SMEs (Small and medium enterprises) involved in R\&D-intensive innovation; 2) increase SMEs participation in public-private partnerships for research; and 3) close gaps within global innovation networks and strengthen linkages between the innovations and marketing processes ${ }^{21,28}$

Government policies which attempt to promote innovation activities of a majority of SMEs through support to early stages of networking may not be sufficient to overcome specific barriers faced by the sub-set of SMEs involved in R\&D-intensive innovation. One answer is to add incentives to collaborative research in existing programmers to support business R\&D, but this favors mostly innovation networks organized around large firms. Another, complementary, approach is to catalyze collaborative R\&D among SMEs through project-based financial incentives ${ }^{29}$. Government also could encourage SMEs participation in Public-Private Partnerships for research and development. Public-Private Partnerships for research sets a framework for the public and the private sectors to join forces in areas where they have complementary interests but cannot act as efficiently alone. Increased participation of SMEs in Public-Private Partnerships is essential for stimulating technological entrepreneurship, ensuring that highly innovative small firms have access to the most fertile sources of knowledge, and linking science-based innovation networks to less R\&Dintensive ones.

Huang ${ }^{30}$ suggests that Taiwan government should implement pre-program and post-program performance evaluation mechanism to avoid poor investment. The primary task is to reconstruct a practical evaluation mechanism for project prioritization ${ }^{9}$. We need to adopt the "Value for Money" approach right away. The method estimates the investment effect of the government in different phases qualitatively and quantitatively as basis for prioritizing investment projects, the process continues into the operational phases of the infrastructure project in order to keep the public informed of the investment returns ${ }^{30}$.

We further suggest that government officials execute broker policies. Broker policies mean that public authorities can support the establishment of links between firms through the creation of platforms for dialogue. The platform also provides support for knowledge-enhancing organization links through 
public-private partnerships, such as incubators, venture capital activities, university laboratories and other research centers. The existence of a technology incubator makes it easier for academic personnel to exploit knowledge-based business ideas, thus lowering the barriers that inhibit direct commercial application of university research results. Technological incubators have assumed a growing role in $R \& D$ research and innovation management, and their importance has not escaped researchers' attention. Incubators, often located between research universities and industry, increasingly mediate the innovation process and their employees, some from academia, others from industry combine practices and professional norms from both worlds in their daily work.

As with any empirical research, this study has limitations. One of them is that the empirical data was collected in only one industry. Different industries have notable differences and the research findings may therefore vary from one country to another. The methodology of this study, DEMATEL research, also presents limitations in terms of the small sample size. Future research can then be conducted to test the adoption factors presented in this study using different methods and larger sample sizes.

\section{Acknowledgments}

This paper is supported by a Grant from the Ministry of Science and Technology (103-2410-H-032 -077 -SSS) in Taiwan for the work from which this paper derived is gratefully acknowledged.

\section{Reference}

1. R. G. Benzle and R. Wink, From agglomerations to technology- and knowledge-driven clusters: aeronautics cluster policies in Europe, Int $J$ Technol Manag. $50(3,4)(2010) 318$.

2. Y. Liu, Y. Zhang, and C. Xu, Analysis of the International Competitiveness of Chinese Medicine Industry Based on the Diamond Model, International Business Research 3 (3) (2010) 165 170.

3. A. Chikán, National and firm competitiveness: a general research model, Competitiveness Review $18(1 / 2)(2008)$ 20-28.

4. T. Dahlberg, N. Mallat, J. Ondrus, and A. Zmijewska,. Past, present and future of mobile payments research: A literature review, Electron
Commer Res Appl. 7 (2) (2008) 165-181.

5. Y. C. Chen and P. F. Hsieh, 4C Diamond Model: Performance Appraisal System Mechanism, Journal of American Academy of Business 10 (2) (2007) 96-106.

6. R. E. Bellm and L. A. Zadeh, Decision-making in a fuzzy environment, Mgt.Sci. 17 (4) (1970) 141164.

7. J. J. H. Liou, L. Yen, and G. H. Tzeng, Building an effective safety management system for airlines, J Air Transp. Manag 4(1) (2007) 20-26.

8. H. E. Yang, C. C. Wu, and K. C. Wang, An Empirical Analysis of Online Game Service Satisfaction and Loyalty, Expert Syst. Appl. 35 (2) (2009) 1816-1825.

9. C. Dögl and D. Holtbrügge, Competitive advantage of German renewable energy firms in Russia - An empirical study based on Porter's diamond, Journal for East European Management Studies 15 (1) (2010) 34-58.

10. J. I. Shieh, H. H. Wu, and K. K. Huang, A DEMATEL method in identifying key success factors of hospital service quality, KnowledgeBased Syst. 23 (3) (2010) 277-282.

11. H. H. Wu and Y. N. Tsai, A DEMATEL method to evaluate the causal relations among the criteria in auto spare parts industry, Appl. Math. Comput. 218 (5) (2011) 2334-2342.

12. W. W. Wu and Y. T. Lee, Developing global managers' competencies using the fuzzy DEMATEL method, Expert Syst. Appl. 32 (2) (2007) 499-507.

13. L.A. Zadeh, Fuzzy sets, 雨8 (3) (1965) 338-353.

14. M.E. Porter, The Competitive Advantage of Nations (The Free Press, NJ, 1998).

15. D. S. Cho, H. C. Moon, and M. Y. Kim. Does one size fit all? A dual double diamond approach to country-specific advantages, Asian Business \& Management 8 (1) (2009) 83-102.

16. M. L. Pettus and M. M. Helms, Government Inclusion in Porter's Diamond: The Case of Argentina, International Journal of Business and Management Science 1(1) (2008) 113-123.

17. T. D. Rojas, National forest economic clusters: A new model for assessing national-forest-based natural resources products and services, Pacific Northwest Research Station Series Paper (2007) $1-40$. 
18. H. C. Lai and J. Z. Shyu, A comparison of innovation capacity at science parks across the Taiwan Strait: the case of Zhangjiang High-Tech Park and Hsinchu Science-based Industrial Park, Technovation 25 (7) (2005) 805-813.

19. S. Sagheer, S. S. Yadav and S. G. Deshmukh, Assessing International Success and National Competitive Environment of Shrimp Industries of India and Thailand with Porter's Diamond Model and Flexibility Theory, Global Journal of Flexible Systems Management 8(1/2) (2007) 31-43.

20. D. N. Ghraith, C. Cowan, and A. Daly, The Market for Organic Milk Products in Ireland: Irish Case Study in Comparison with Danish and Austrian Cases, Irish Journal of Management 25(2) (2004) 121-138.

21. Y. Y. Wong, T. E. Maher, J. L .H. Wang, and F. Long, Exploring Taiwan's competitive advantages: Present and future, Management Research News 24(6/7) (2001) 17-24.

22. V. M. R. Tummala, H. Y. H. Lee, and R. C. M. Yam, Strategic alliances of China and Hong Kong in manufacturing and their impact on global competitiveness of Hong Kong manufacturing industries, Integrated Manufacturing Systems 11(6) (2000) 370.

23. H. H. Wu, H. K. Chen, and J. I. Shieh, Evaluating performance criteria of Employment Service Outreach Program personnel by DEMATEL method, Expert Syst. Appl. 37(7) (2010) 52195223.

24. W.W. Wu, Choosing knowledge management strategies by using a combined ANP and DEMATEL approach, Expert Syst. Appl. 35(3) (2008) 828-835.

25. C. Y. Huang, J. Z. Shyu, and G. H. Tzeng, Reconfiguring the innovation policy portfolios for Taiwan's SIP Mall industry, Technovation 27(12) (2007) 744-765.

26. G. H. Tzeng, C. H. Chiang, and C. W. Li, Evaluating intertwined effects in e-learning programs: A novel hybrid MCDM model based on factor analysis and DEMATEL, Expert Syst. Appl. 32(4) (2007) 1028-1044.

27. S. Sagheer, S. S. Yadav, and S. G. Deshmukh, Assessing International Success and National Competitive Environment of Shrimp Industries of India and Thailand with Porter's Diamond Model and Flexibility Theory, Global Journal of Flexible Systems Management 8(1/2) (2007) 31-43.

28. V. M .R. Tummala, H. Y. H. Lee, and R. C. M. Yam, Strategic alliances of China and Hong Kong in manufacturing and their impact on global competitiveness of Hong Kong manufacturing industries, Integrated Manufacturing Systems 11(6) (2000) 370.

29. M. L. Pettus and M. M. Helms,. Government Inclusion in Porter's Diamond: The Case of Argentina, International Journal of Business and Management Science 1(1) (2008) 113-123.

30. H. Huang, Challenges and Opportunities for Infrastructure Development in Taiwan, (Taiwan Institute of Economic research, 2008). 